\title{
Assistência ventricular direita facilitando revascularização de vasos posteriores com coraçẳo batendo: experiência clínica inicial
}

\author{
Leonardo Esteves LIMA*, Nestor SABATOVICS*, Maria Cristina REZENDE*, Pedro PANIAGUA*, \\ Itacir A. FRANCESCHINI*, Ricardo CARRANZA*, Candido GOMES*, Dilma LOPES*, \\ André Esteves LIMA*
}

RBCCV 44205-513

Lima L E, Sabatovics N, Rezende M C, Paniagua P, Franceschini I A, Carranza R, Gomes C, Lopes D, Lima A E - Assistência ventricular direita facilitando revascularização de vasos posteriores com coração batendo: experiência clínica inicial. Rev Bras Cir Cardiovasc 2000; 15(4): 279-84.

RESUMO: Na revascularização do miocárdio com o coração batendo, a exposição das artérias posteriores durante a esternotomia pode levar à deterioração hemodinâmica necessitando um suporte inotrópico. Estudos experimentais recentes demonstraram o benefício hemodinâmico da assistência ventricular direita (AVD).

Objetivo: Avaliar os efeitos hemodinâmicos da AVD durante a manipulação cardíaca para expor as artérias coronárias posteriores, determinando seu efeito em: restaurar a hemodinâmica, aumentando a exposição anastomótica e diminuindo o uso de inotrópicos.

Casuística e Métodos: Onze pacientes (6 homens / 5 mulheres), com idade média de 63,3 anos (49-74) foram revascularizados utilizando AVD. Todos tiveram pelo menos 1 ramo da artéria circunflexa (CX) revascularizado. A parede anterior foi revascularizada sistematicamente sem circulação extracorpórea (CEC) ou AVD. A artéria coronária direita (CD) e o ramo da CX foram completados em AVD. A pressão arterial média (PAM), a pressão média da artéria pulmonar (PAP) e o débito cardíaco (DC) foram registrados durante as anastomoses CD e CX.

Resultados: Os dados foram registrados da seguinte maneira: 1) base em normoposição; 2) CX em posição AVD Off; 3) CX em posição AVD On; 4) CD em posição AVD Off; 5) CD em posição AVD On.

$\begin{array}{llllll} & 1 \mathrm{BASE} & 2 \mathrm{CX} \text { AVD off } & 3 \mathrm{CX} \text { AVD on } & 4 \mathrm{CD} \text { AVD off } & 5 \mathrm{CD} \text { AVD on } \\ \text { PAM } & 66 \mathrm{mmHg} & 44 \mathrm{mmHg} & 68 \mathrm{mmHg} & 63 \mathrm{mmHg} & 81 \mathrm{mmHg} \\ \text { PAP } & 18 \mathrm{mmHg} & 18 \mathrm{mmHg} & 17 \mathrm{mmHg} & 19 \mathrm{mmHg} & 18 \mathrm{mmHg} \\ \text { DC } & 5,2 \text { litros } & 2,1 \text { litros } & 4,4 \text { litros } & 3,3 \text { litros } & 4,9 \text { litros }\end{array}$

Conclusões: A AVD suporta e facilita a revascularização sem CEC para as artérias coronárias posteriores, restaurando a hemodinâmica, possibilitando melhor exposição para as anastomoses e, aparentemente, reduzindo a necessidade de uso de inotrópicos. Estudos prospectivos randomizados são necessários para confirmar esta experiência inicial.

DESCRITORES: Revascularização miocárdica, métodos. Hemodinâmica, fisiopatologia. Circulação extracorpórea. Função ventricular direita, fisiologia. Ventrículo cardíaco. Vasos coronários, cirurgia. Coração auxiliar.

Trabalho realizado no Cardiocentro, Hospital Santa Lúcia. Brasília, DF, Brasil e InCor, Hospital Anchieta, Taguatinga, DF, Brasil. Apresentado ao 27으 Congresso Nacional de Cirurgia Cardíaca. Rio de Janeiro, 23 a 25 de março de 2000.

* Do Cardiocentro, Hospital Santa Lúcia e Incor, Hospital Anchieta.

Endereço para correspondência: Leonardo Esteves Lima. SQS 111, Bloco G, apto. 604. Brasília, DF, Brasil. CEP: 70734-070. Tel: (061) 245-3344.

e-mail: cardiocentro@ambr.com.br 
Lima L E, Sabatovics N, Rezende M C, Paniagua P, Franceschini I A, Carranza R, Gomes C, Lopes D, Lima A E - Assistência ventricular direita facilitando revascularização de vasos posteriores com coração batendo: experiência clínica inicial. Rev Bras Cir Cardiovasc 2000; 15(4): 279-84.

TABELA 1

\section{DADOS PRÉ-OPERATÓRIOS DOS PACIENTES}

\begin{tabular}{lll}
\hline Sexo & Masculino 6 & Feminino 5 \\
\hline Idade & $63.3(49-74)$ & \\
CF (NYHA) & $3-4$ \\
Reoperação & 1 paciente \\
\hline Angina instável & 3 pacientes \\
№ de vasos doentes & 34 \\
LTCE & 3 pacientes \\
FEVE & 9 pac. $>50 \%, 1$ pac. $30-40 \%$ \\
\hline
\end{tabular}

CF: classe funcional; LTCE: lesão de tronco comum esquerdo; FEVE: fração de ejeção de ventrículo esquerdo

\section{INTRODUÇÃO}

A revascularização do miocárdio (RM) sem circulação extracorpórea (CEC) e através de uma esternotomia mediana tem sido utilizada desde o início da RM. Os benefícios de se realizar a RM sem CEC já foram bem documentados na literatura e incluem baixa morbi-mortalidade, redução de custos e diminuição de transfusões sangüíneas (1-3). Recentemente, esta estratégia cirúrgica tem ganhado maior interesse para revascularização de múltiplos vasos $(1,2,4)$ e tem sido praticada em todo o mundo. Em nossa experiência pessoal, $90 \%$ de todos os nossos pacientes que necessitaram RM em 1999 foram feitos sem CEC, mesmo assim, ainda restam $10 \%$ dos casos, nos quais a CEC é necessária; e isso principalmente por deterioração da hemodinâmica e/ou difícil acesso e visibilização de alguns vasos posteriores.

Recentemente, estudamos as mudanças hemodinâmicas durante a RM sem CEC e achamos que, mesmo durante a exposição da artéria interventricular anterior, existe uma diminuição do débito cardíaco (DC), e esta diminuição tende a ser mais importante quando o coração é deslocado para realização de pontes em vasos posteriores como a artéria circunflexa $(C X)$ e a interventricular posterior. Este deslocamento causa, em alguns casos, instabilidade hemodinâmica durante a RM com coração batendo.

GRUNDEMAN et al. (5) e JANSEN et al. (6) relataram, em animais e pacientes, que a exposição de vasos posteriores através do deslocamento do coração leva à diminuição da pressão arterial, basicamente devido à disfunção ventricular direita causada pela interferência mecânica na expansão diastólica. Em animais, a assistência direita normalizou o volume de ejeção e a pressão arterial média (PAM) aumentado a pré-carga ventricular.
Os objetivos deste estudo são avaliar as modificações hemodinâmicas durante a manipulação cardíaca feita para expor artérias coronárias posteriores e determinar os efeitos da assistência ventricular direita (AVD) em: restaurar a hemodinâmica, aumentar a exposição para realização das anastomoses e reduzir a necessidade de uso inotrópicos.

\section{CASUÍSTICA E MÉTODOS}

\section{Pacientes}

Onze pacientes foram submetidos a RM com o coração batendo e AVD. As operações foram realizadas pela mesma equipe trabalhando em dois hospitais (Hospital Santa Lúcia e Hospital Anchieta, DF - Brasil). Todos os pacientes eram candidatos à operação de RM com o coração batendo, com pelo menos um ramo da artéria circunflexa a ser tratado. Pacientes com instabilidade elétrica foram excluídos. Os dados préoperatórios dos pacientes são apresentados na Tabela 1.

\section{Técnica Cirúrgica}

Raquianalgesia com morfina foi feita em todos os casos. A anestesia foi induzida com sulfentanil e propofol e mantida com sulfentanil, propofol e uma mistura de óxido nítrico com brometo de pancurônio. No início da intervenção, uma infusão contínua de nitroglicerina e papaverina foi administrada; e volume infundido de acordo com a necessidade. As artérias torácicas internas e/ou radiais foram preparadas semi-esqueletizadas como descrito por 
Lima L E, Sabatovics N, Rezende M C, Paniagua P, Franceschini I A, Carranza R, Gomes C, Lopes D, Lima A E - Assistência ventricular direita facilitando revascularização de vasos posteriores com coração batendo: experiência clínica inicial. Rev Bras Cir Cardiovasc 2000; 15(4): 279-84.

CALAFIORE et al. (7). Os enxertos foram seccionados após heparinização geral $(3 \mathrm{mg} / \mathrm{kg}$ de peso). As artérias a serem revascularizadas foram expostas segundo a estratégia proposta por BERGSLAND et al. (8). As artérias anteriores (interventricular anterior e diagonais) foram tratadas com coração batendo sem (sem AVD) utilizando estabilizadores mecânicos de sutura. Os vasos posteriores (CX e interventricular posterior) foram expostos, uma vez que o sistema de AVD estava instalado: o paciente foi colocado em posição de Trendelenburg e basculado para direita. O estabilizador foi colocado na melhor posição possível, sem levar em conta a diminuição da PAM e/ou DC. Nenhuma droga inotrópica foi utilizada, com exceção de 2 pacientes que já estavam em uso de inotrópicos antes da operação. Uma vez que a melhor PAM e DC eram registrados (em geral 2 a 5 minutos após à estabilização), a AVD era iniciada com um débito mínimo, o qual foi aumentado progressivamente até atingir a mais elevada PAM e/ou DC ou restaurar completamente a hemodinâmica. No final da anastomose, a AVD era parada e o coração colocado em sua posição anatômica. Todas as anastomoses foram realizadas com sutura contínua. O sangue perdido durante a operação foi recuperado e reinfundido utilizando uma máquina de autotransfusão. A protamina foi feita na dose de $1: 1$ e os pacientes fechados de modo habitual.

\section{O Sistema A-Med}

A bomba inclui a cânula A-Syst Coaxial em "J", um cabo rotor ligado à bomba centrífuga ASyst, um motor e um console de controle. A cânula coaxial em "J" é posicionada através da valva tricúspide e a cânula interna é avançada de 10 a $15 \mathrm{~cm}$ até ser posicionada na artéria pulmonar através da valva pulmonar, próximo à sua bifurcação. O posicionamento da cânula é verificado através de palpação direta do coração. A bomba centrifuga miniatura é posicionada no campo cirúrgico e conectada à cânula. $\mathrm{A}$ bomba é conectada ao motor o qual, por sua vez, é conectado ao console; e estes dois se encontram fora do campo (Figura 1).

\section{Dados Hemodinâmicos}

Para avaliar a mudança hemodinâmica durante a manipulação cardíaca, foram medidas: PAM, PAP e DC através de ecodopplercardiografia transesofágico, durante a operação. Os dados e complicações peri-operatórias e pósoperatórias foram registrados até a alta dos pacientes.

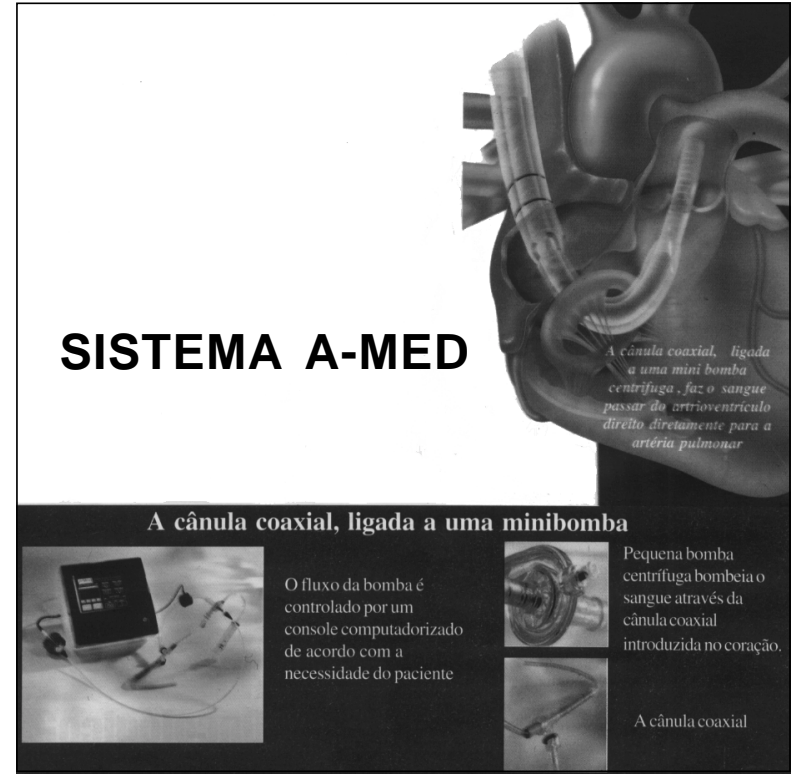

Fig 1- Sistema A-MED e assistência direita

\section{RESULTADOS}

A operação foi um sucesso em todos os pacientes. E a melhor visibilização das artérias profundas foi possível em todos os casos, sendo o estabilizador reposicionado uma vez em AVD, meIhorando a exposição. A maioria dos pacientes teve pontes arteriais (89\% dos enxertos), em um total de 34 anastomoses realizadas, perfazendo uma média de 3 anastomoses por paciente. A Tabela 2 mostra a evolução da PAM, PAP e DC durante a manipulação cardíaca e também o fluxo médio necessário para restaurar uma parte ou completamente a hemodinâmica base.

O tempo médio de UTI foi de $26 \pm 8$ horas e de hospitalização de $6 \pm 1$ dias. O sangramento médio foi de $350 \pm 123 \mathrm{ml}$. Somente 2 pacientes necessitaram transfusão sangüínea. Nenhum paciente teve infarto do miocárdio, insuficiência renal e/ou complicações respiratórias. Apenas 2 pacientes necessitaram inotrópicos após a operação por mais de 6 horas, e estes já estavam em uso de drogas no préoperatório. Não houve complicações devido ao uso da AVD. Todos os pacientes tiveram alta hospitalar.

\section{COMENTÁRIOS}

O desenvolvimento dos estabilizadores de sutura permitiu aos cirurgiões realizarem RM cada vez mais desafiadoras. No entanto, em alguns casos a dificuldade de acesso a vasos posteriores do coração, mantendo uma boa estabilidade hemodinâmica 
Lima L E, Sabatovics N, Rezende M C, Paniagua P, Franceschini I A, Carranza R, Gomes C, Lopes D, Lima A E - Assistência ventricular direita facilitando revascularização de vasos posteriores com coração batendo: experiência clínica inicial. Rev Bras Cir Cardiovasc 2000; 15(4): 279-84.

TABELA 2

\begin{tabular}{llcccc}
\hline \multicolumn{7}{c}{ EVOLUÇÃO DA PAM, PAP, DC FLUXO MÉDIO DA BOMBA } \\
\hline $\begin{array}{c}\mathbf{1} \mathbf{P} \\
\text { Básica }\end{array}$ & $\begin{array}{c}\mathbf{2} \mathbf{C X} \\
\text { Bomba Off }\end{array}$ & $\begin{array}{c}\mathbf{3} \mathbf{C X} \\
\text { Bomba On }\end{array}$ & $\begin{array}{c}\mathbf{4} \text { Rvp } \\
\text { Bomba Off }\end{array}$ & $\begin{array}{c}\mathbf{5} \text { Rvp } \\
\text { Bomba On }\end{array}$ \\
\hline PAM & $66 \mathrm{mmHg}$ & $44 \mathrm{mmHg}$ & $68 \mathrm{mmHg}$ & $63 \mathrm{mmHg}$ & $81 \mathrm{mmHg}$ \\
DC & $5.6 \mathrm{l} / \mathrm{min}$ & $2.1 \mathrm{l} / \mathrm{min}$ & $4.4 \mathrm{l} / \mathrm{min}$ & $3.3 \mathrm{l} / \mathrm{min}$ & $4.7 \mathrm{l} / \mathrm{min}$ \\
FMB & - & - & $2.1 \mathrm{l} / \mathrm{min}$ & - & $1.5 \mathrm{l} / \mathrm{min}$ \\
PAP & $18 \mathrm{mmHg}$ & $18 \mathrm{mmHg}$ & $17 \mathrm{mmHg}$ & $19 \mathrm{mmHg}$ & $18 \mathrm{mmHg}$ \\
\hline
\end{tabular}

e visibilidade, ainda persiste. Este sistema de assistência circulatória miniaturizado parece manter a estabilidade hemodinâmica necessária durante a operação de coração batendo. Em alguns casos, sentimos que não seria possível realizar a operação sem este sistema de suporte. Dois destes pacientes tinham angina instável e estavam em uso de inotrópicos antes da operação.

A RM com o coração batendo tem várias vantagens, entre elas: menor ativação dos fatores inflamatórios, ausência de consumo importante de fatores de coagulação, diminuição do número de transfusões sangüíneas, todos os quais são mais importantes com o uso de CEC. Este sistema parece diminuir a área de contato com o sangue, mas ainda necessita de melhor avaliação para poder provar a possibilidade de reduzir a resposta inflamatória.

O resultado mais importante deste estudo parece ser a restauração do DC associado ao aumento do débito da bomba e, na maioria dos casos, sem necessidade de uso de suporte inotrópico. Neste estudo, manobras tradicionais como a posição de Trendelemburg, a infusão de líquido e a estratégia de exposição dos vasos foram utilizadas com a intenção de restaurar a PAM e o DC, mas mesmo assim, a hemodinâmica só foi restaurada com o uso de AVD.

Sabemos que em alguns casos o uso de inotrópicos restaura a hemodinâmica. No momento, não sabemos se o uso de inotrópicos é mais benéfico do que a AVD. A questão da necessidade de se realizar uma assistência esquerda, direita ou biventricular durante RM com coração batendo pode ser evocada. GRUNDEMAN et al. (5) demonstraram que a assistência esquerda falhou em restaurar a circulação sistêmica, e LONN et al. (9) demonstraram que a assistência esquerda seria mais eficaz para pacientes com insuficiência ventricular esquerda. No nosso estudo, um paciente tinha menos que $25 \%$ de fração de ejeção e, mesmo assim, dois ramos da artéria CX foram tratados, sem uso de qualquer inotrópico. Se seguirmos a evolução da PAM (Tabela 2) durante o uso da AVD, verifica-se que a mesma foi restaurada em $100 \%$, mas quanto ao DC, apenas $90 \%$ foi restaurado. Isto pode, provavelmente, ser explicado por um certo grau de insuficiência esquerda causada pela aplicação do estabilizador e pelo deslocamento do coração. A minibomba centrífuga usada para a AVD foi de uso fácil e intuitivo. A cânula coaxial foi de fácil inserção em todos os casos.

\section{CONCLUSÃO}

Concluímos que o uso do sistema A-MED como AVD facilitou a realização de $R M$ com coração batendo para vasos posteriores, restaurando a hemodinâmica de base, melhorando a visualização da região anastomótica e aparentemente reduzindo a necessidade de suporte inotrópico. Apenas um pequeno número de casos foi realizado com este sistema, de modo que um número maior precisa ser estudado para poder confirmar esta experiência inicial. 
Lima L E, Sabatovics N, Rezende M C, Paniagua P, Franceschini I A, Carranza R, Gomes C, Lopes D, Lima A E - Assistência ventricular direita facilitando revascularização de vasos posteriores com coração batendo: experiência clínica inicial. Rev Bras Cir Cardiovasc 2000; 15(4): 279-84.

RBCCV 44205-513

Lima L E, Sabatovics N, Rezende M C, Paniagua P, Franceschini I A, Carranza R, Gomes C, Lopes D, Lima A E - Right heart support to facilitate beating heart coronay surgery to posterior vessels: early clinical experience. Rev Bras Cir Cardiovasc 2000; 15(4): 279-84.

ABSTRACT: Objectives: During coronary surgery without CPB, exposure of posterior vessels via sternotomy can cause deterioration of cardiac hemodynamics, requiring inotropic drug support. Recent animal experiments demonstrate the hemodynamic benefit of right heart support (RHS) with the A-MED system. The aim of this study is to evaluate the hemodynamic effect of RHS in: restoring hemodynamics, increasing anastomotic exposure and reducing inotropic requirements.

Material and Methods: Eleven patients received coronary revascularization with the A-MED RHS device. All had at least 1 circumflex branch bypassed. The anterior wall was systematically bypassed off pump without RHS. The right coronary artery $(R C A)$ and the obtuse coronary artery $(O M)$ were completed utilizing RHS. Mean arterial pressure (MAP), mean pulmonary arterial pressure (PAP), cardiac output (CO) and the average pump flow (APF) were recorded during the OM and RCA bypass.

Results: Elective beating heart coronary artery graft (CABG) was successfully accomplished in 11 patients with RHS. Data measurements recorded showed the improved hemodynamic recovery for OM and RCA bypass with RHS. The MAP increased from 44 to $63 \mathrm{mmHg}(\mathrm{OM})$ and from 63 to $81 \mathrm{mmHg}(\mathrm{RCA})$. No device-related patient incidents occurred. All 11 patients were discharged to their homes.

Conclusions: The AMED system as RHS support facilitated coronary bypass without CPB to posterior vessels, restoring hemodynamics, providing better exposure to anastomic sites and apparently reducing the need for inotropic support.

DESCRIPTORS: Myocardial revascularization, methods. Hemodynamics, physiopathology. Extracorporeal circulation. Ventricular function, right, physiology. Heart ventricle. Coronary vessels, surgery. Heart-assist devices.

\section{REFERÊNCIAS BIBLIOGRÁFICAS}

1 Benetti F J, Naselli G, Wood M, Geffner L - Direct myocardial revascularization without extracorporeal circulation: experience in 700 patients. Chest 1991; 100: 312-6.

2 Buffolo E, de Andrade C S, Branco J N, Teles C A, Aguiar L F, Gomes W J - Coronary artery bypass grafting without cardiopulmonary bypass. Ann Thorac Surg 1996; 61: 63-6.

3 Westaby S - Coronary surgery without cardiopulmonary bypass. Br Heart $J$ 1995; 73: 203-5.

4 Calafiore A M, Teodori G, Di Giammarco G et al. Multiple arterial conduits without cardiopulmonary bypass: early angiographic results. Ann Thorac Surg 1999; 67: 450-6.

5 Grundeman P F, Borst C, Verlaan C W, Meijburg H, Moues C M, Jansen E W - Exposure of circumflex branches in the tilted, beating porcine heart: echocardiographic evidence of right ventricular deformation and the effect of right or left heart bypass. J Thorac Cardiovasc Surg 1999; 118: 316-23.

6 Jansen E W, Borst C, Lahpor J R et al. - Coronary artery grafting without cardiopulmonary bypass using the octopus method: results in the first one hundred patients. J Thorac Cardiovasc Surg 1998; 116: $60-7$.
7 Calafiore A M, Di Giammarco G, Luciani N, Maddestra N, Di Nardo E, Angelini R - Composite arterial conduits for a wider arterial myocardial revascularization. Ann Thorac Surg 1994; 58: 185-90.

8 Bergsland J, Karamanoukian H L, Soltoski P R, Salerno T A - "Single suture" for circumflex exposure in offpump coronary artery bypass grafting. Ann Thorac Surg 1999; 68: 1428-30.

9 Lonn U, Peterzen B, Carnstam B, Casimir-Ahn H - Beating heart coronary surgery supported by an axial blood flow pump. Ann Thorac Surg 1999; 67: 99-104.

\section{Discussão (transcrições de fita gravada)}

\section{DR. LUIZ ANTÔNIO RIVETTI}

São Paulo, SP

Parabenizo o Dr. Leonardo pelo trabalho de alta qualidade. Nós que realizamos cirurgia sem circulação extracorpórea em quase metade dos nossos pacientes há 17 anos, sabemos que existem alguns casos que, mesmo abordando apenas as artérias coronárias inferiores e anteriores do coração, não podemos nem olhar para o coração que ocorre alteração hemodinâmica. Em cerca de 15\% dos pacientes, não pudemos nem colocar uma compressa ou posicionar o pericárdio com tração para melhor 
Lima L E, Sabatovics N, Rezende M C, Paniagua P, Franceschini I A, Carranza R, Gomes C, Lopes D, Lima A E - Assistência ventricular direita facilitando revascularização de vasos posteriores com coração batendo: experiência clínica inicial. Rev Bras Cir Cardiovasc 2000; 15(4): 279-84.

visibilizar a artéria coronária; tivemos que operar em uma condição precária, mas foi possível. Então, imaginem a tentativa de realizar revascularização nas artérias coronárias posteriores do coração, nesses pacientes. Acredito que para quem se propuser a realizar $100 \%$ das revascularizações sem circulação extracorpórea, este dispositivo é mandatório. A primeira extracorpórea realizada no Brasil pelo Dr. Filipozzi, para correção de uma estenose pulmonar, e sabemos que o desvio direito aumenta a pré-carga e o desvio esquerdo diminui esta. Gostaria de perguntar como você imagina que os corações com fração de ejeção muito baixa vão se comportar, aumentando a pré-carga e piorando a condição subendocárdica desses pacientes? Sob ponto de vista técnico, como vai se comportar o ventrículo frente ao aumento da pré-carga, a visibilização da artéria coronária não vai ficar comprometida? É lógico que os estabilizadores auxiliam muito. Só numa fase bem inicial, tivemos a preocupação de diminuir a freqüência cardíaca daqueles pacientes, mas a partir de um determinado momento nós não nos incomodamos mais com a freqüência cardíaca elevada porque, as vezes, a distorção diminui o volume de ejeção e, consequentemente, aquela freqüência tem que ser maior mesmo, para ter um débito estável. Concluindo, acho que este dispositivo é fundamental para quem queira fazer revascularização do miocárdio sem circulação extracorpórea, o que eu chamaria de uma cirurgia moderadamente invasiva ou de uma miniextracorpórea.

\section{DR. Waldir jazbik}

Rio de Janeiro, $R J$

No shunt do Dr. Domingos não é necessária uma saturação tão alta e nós sabemos que o paciente ventilando pode adquirir um $\mathrm{PO}_{2}$ alto, assim não seria mais simples injetar da aurícula direita ou na aorta ou na artéria femoral? Isto poderia aliviar o coração direito e não sobrecarregar o esquerdo. Gostaria de saber a opinião do autor sobre este aspecto.

\section{DR. FABIO B. JATENE \\ São Paulo, $S P$}

Gostaria de cumprimentar o Dr. Leonardo e fazer alguns comentários. Eu, por iniciativa do Prof. Enio Buffolo e do próprio Dr. Leonardo, tive a experiência em nosso grupo de fazer 8 casos com este sistema. Acho que este é um sistema eficiente, fácil de ser utilizado, entretanto, há a necessidade de um trabalho comparativo utilizando este sistema em um grupo de pacientes e em outro grupo usando o ponto do Dr. Ricardo Lima ou outro mecanismo. É preciso avaliar, também, pontes para as paredes lateral e inferior, tipo ventricular ou descendente posterior, para dizer se realmente este sistema é útil ou não. Tive a oportunidade de fazer este mesmo comentário para os representantes do laboratório AMed. Acredito que o próximo passo será tentar comparar os métodos para verificar se, realmente, em relação a débito cardíaco, comportamento hemodinâmico, drogas, entre outros parâmetros, este dispositivo traz benefícios.

\section{DR. Lima \\ (Encerrando)}

Com relação à primeira pergunta do Dr. Rivetti, sobre a pré-carga e ventrículos ruins, gostaria de registrar que nós já realizamos este tipo de assistência em ventrículos ruins. Tive a oportunidade de participar de, pelo menos, 3 ou 4 casos onde a fração de ejeção era inferior a 25\%; e quando luxamos este tipo de ventrículo, não só ocorre insuficiência ventricular direita, mas também, esquerda. Nesses casos, 3 foram realizados e 2 necessitaram de apoio de drogas inotrópicas, ou seja, o suporte ventricular esquerdo foi dados através de drogas e o direito através da bomba. Esta é uma resposta parcial, mas será que todos podem ser realizados assim? Eu creio que não; alguns pacientes, provavelmente, irão necessitar de circulação extracorpórea. Com relação ao aspecto técnico de visibilização das artérias, é verdade que a pré-carga aumentando você poderia pensar que isto diminui a visibilização, mas quando fazemos a ponte sem circulação extracorpórea, normalmente, comprometemos um pouco, não comprimimos tanto porque temos uma boa hemodinâmica. Com a assistência ventricular direita, sabemos que isto não existe mais, ou existe muito menos, e que a compressão pode ser muito maior e a exposição melhor, independente de toda essa pré-carga. Com relação às drogas, aparentemente para esses que não estão de acordo para utilização de drogas, este sistema diminuiria o uso de drogas na maior parte dos casos. Com relação ao comentário do Dr. Waldir Jazbik, da assistência da aurícula direita para aorta ou artéria femoral, sem dúvida alguma isto pode ser realizado, mas seria provavelmente um pouco mais invasivo porque estaríamos tocando nestes vasos. No meu ponto de vista, a perfusão através da aorta é muito mais invasiva do que na artéria pulmonar. Com relação ao comentário do Dr. Fabio Jatene sobre a necessidade de realização de um trabalho comparativo, acho que é uma verdade. Um grupo da Alemanha já está iniciando um estudo comparativo com 50 casos e acredito que no próximo ano teremos várias respostas, que permitirão avaliarmos se iremos ou não utilizar esse sistema. 